\title{
CODE: A Description Language for Wireless Collaborating Objects
}

\author{
"Raluca Marin-Perianu, Hans Scholten, Paul Havinga \\ Department of Computer Science, University of Twente \\ P.O. Box 217, 7500 AE Enschede \\ \{r.s.marinperianu, j.scholten, p.j.m.havinga\}@utwente.nl
}

\begin{abstract}
This paper introduces CODE, a Description Language for Wireless Collaborating Objects (WCOs), with the specific aim of enabling service management in smart environments. WCOs extend the traditional model of wireless sensor networks by transferring additional intelligence and responsibility from the gateway level to the network. WCO are able to offer complex services based on cooperation among sensor nodes. CODE provides the vocabulary for describing the complex services offered by WCO. It enables description of services offered by groups, on-demand services, service interface and sub-services. The proposed methodology is based on XML, which is widely used for structured information exchange and collaboration. CODE can be directly implemented on the network gateway, while a lightweight binary version is stored and exchanged among sensor nodes. Experimental results show the feasibility and flexibility of using CODE as a basis for service management in $W C O$.
\end{abstract}

\section{INTRODUCTION}

Wireless Sensor Networks is an emerging technology that has led to extensive studies concerning the new challenges that researchers and programmers have to overcome, such as energy efficiency, scarce computing and storage resources, unreliable communication, harsh environments. Therefore, most of the initiatives have focused on tackling these difficulties rather than providing rich functionality within complex world scenarios. Consequently, applications usually utilize sensor nodes for monitoring or tracking purposes within a static pattern: collect data, (optionally) perform some in-network processing and forward the results to a central system. Wireless Collaborating Objects (WCO) is a paradigm that extends the traditional model of wireless sensor networks by transferring intelligence and responsibility to sensor nodes. Nevertheless, a single sensor node is limited in terms of hardware performance and the energy available. Under these conditions, the overall performance of the network can only be improved through cooperation among sensors.

The vision of WCO is that nodes self-organize into dynamic groups and offer services based on the capabilities of each member. Groups are formed either as a result of a system configuration or simply "on-demand" as a result of a service request. The latter case is more dynamic, as groups are formed based on context information and are dissolved once the service is accomplished.

Another idea of WCO is the dynamic nature of service deployment in the network. New services can be constructed from existing ones, while other services can be deleted from the network. The service discovery protocol needs to be adapted to such a highly dynamic environment and to enable service composition through a flexible vocabulary.

In this paper, we propose the CODE service description language as a means of describing collaborating objects and the services they offer. It can be used to support methods and tools to achieve service management in WCO. In particular, CODE allows for extensible descriptions of the service interface, sub-services, service requirements, consuming entities and service attributes. The contributions of our work are as follows:

1. We identify the basic components of service management for WCO.

2. We propose CODE, a generic and extensible method for describing dynamic entities and services in WCO.

3. We show that the binary version of CODE is feasible of being stored and processed on sensor nodes.

The rest of this paper is organized as follows. Section 2 presents a scenario that motivates our approach for service management for WCO. Section 3 describes the basic components of service management and the role of the service description language. Section 4 covers the description of services and groups in WCO. Section 5 demonstrates the feasibility of binary CODE to work on sensor nodes. Section 6 discusses related work and Section 7 presents a summary and future work.

\section{Motivating SCEnARIO}

We present an example that illustrates most of the concepts of service management for WCO. The scenario concerns the process of transporting goods from providers to consumers. The products are placed on shelves which are fitted to rolling carts. Each cart is equipped with a wireless sensor node, termed a micronode. The nodes on the carts communicate with each other wirelessly, creating an ad-hoc network. They are powered by batteries, so energy saving is a major objective. Sensors called piconodes are placed on each shelf 


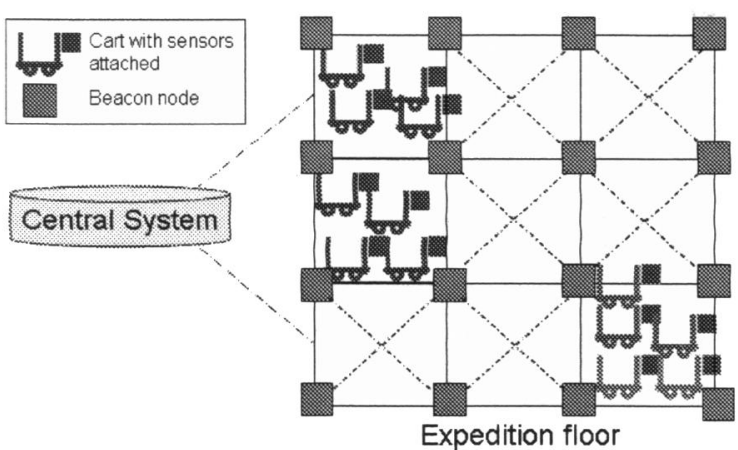

Fig. 1. Process diagram.

Carts gather on allocated grid rectangles within the expedition floor.
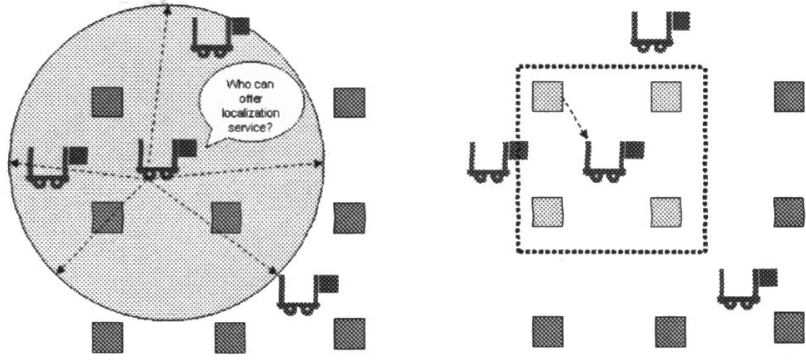

Fig. 2. Localization service.

The cart broadcasts a message asking for localization service. Beacons that hear the broadcast organize into a group and deliver the service to the cart. of the cart for a precise control of environmental conditions when the delicate and perishable goods (such as flowers) are being transported.

In this scenario, we focus on the expedition floor, which is a place used by the transport company for the shipment process. A large grid is painted on the expedition floor and each cell of the grid is associated with a certain shop. Loaded carts arrive on the expedition floor and are placed depending on the shop they are assigned to. The carts belonging to one shop are grouped together and occupy a part of, one or more adjacent grid cells, depending on the number of carts.

A fixed infrastructure of sensor nodes is placed uniformly in the grid on the expedition floor. These nodes are referred to as beacons and will facilitate the localization process. They are connected to the mains electrical supply, so energy saving is not a priority. Beacons will also act as communication gateways between the cart nodes and a central coordinator, called Central System (CS), which is a computer in charge of coordinating all actions on the expedition floor.

CS informs the beacons about: (1) the assignment of carts to cells, (2) the environmental conditions that have to be observed and (3) the deadlines for carts to gather. The beacons actively calculate positions of the carts and verify correct gathering, environmental conditions and deadlines. The events and results of the monitoring process are transmitted back to CS.

Figure 1 illustrates the process. The filled carts are placed on the expedition floor on certain grid cells, which are delimited by beacon nodes. Groups of carts placed within adjacent cells are then transported together in the same trailer.

\section{A. Group functionality}

As the fixed infrastructure attached to the expedition floor is supplied with mains power, it is desirable that it should take over as much as possible of the computation and monitoring services present in the network. The set of beacons which form the fixed infrastructure organize into groups for providing the following services:

1. localization service, for the carts placed on the expedition floor.

2. shop monitoring service, for the Central System.
The second service is provided by groups of beacons formed as a result of a configuration message from $\mathrm{CS}$, which assigns the shops and carts to be monitored and decides which are the members of the groups. The first service is offered ondemand and is provided by dynamically formed groups.

\section{B. On-demand services}

Services can be offered on-demand as a result of a service discovery message. Figure 2 shows an example of how beacons dynamically organize into a group that deliver a localization service for a nearby cart. On the left-hand side of the picture, one cart broadcasts a service discovery message. Beacons that receive the broadcast organize into a group that is able to deliver the localization service, based on the information owned by each member. On the right-hand side of the picture, the beacons that become members of the group are highlighted.

\section{Service composition}

The monitoring service performed by the group of beacons assigned to a shop is composed of a set of sub-services, concerning monitoring of carts gathering, monitoring of environmental conditions and verification of deadlines. Monitoring of the gathering carts uses the location information service offered by each cart belonging to the shop being monitored. Carts, in turn, use the localization service offered by groups of beacons from the neighbourhood. The monitoring of environmental conditions service uses the humidity, light and temperature services, which further rely on the environmental services offered by each cart. Figure 3 shows an example of service composition for monitoring the carts on the expedition floor. It can be observed that, taking advantage of the existing services, complex ones can be built. Consequently, the level of functionality increases.

\section{Service Management}

The above scenario points out the new concept of service management for WCO. Services are offered not only by single devices, but also by groups. Service offer is not fixed, but it changes depending on the context. Simple services can be combined resulting in more complex ones, and this process 


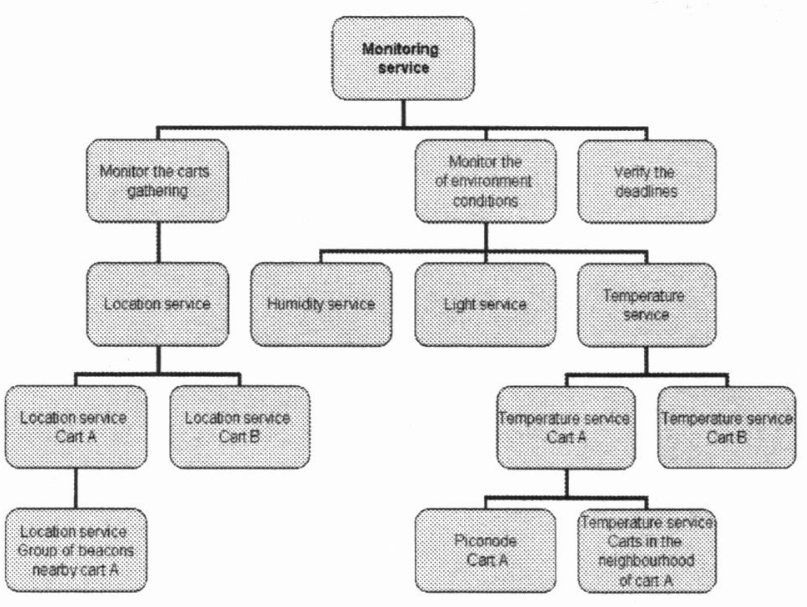

Fig. 3. Composition of services.

Each complex service is composed of a number of sub-services.

can be repeated. The outcome is a whole hierarchy of services.

We call service management the framework of service manipulation in WCO. It consists of the following modules:

- Service deployment. Services are deployed on the network nodes in WCO.

- Service composition. Complex services are constructed from the existing ones.

- Service discovery. Discovery is the action of finding a service provider for a requested service.

- Service policy. Service policy controls the access rights of the service consumer to the requested service.

- Service usage. Service usage is the process of making use of the available service.

All the components of service management are directly dependent on an additional ingredient: Service description.

Deployment is assisted through description of service requirements. Services can be mapped on specific nodes of the network, or on entities which fulfil certain characteristics. The service requirements specify the desired features of service providers. Following the scenario explained in Section 2 , the temperature service is deployed only on carts fitted with a piconode that measures temperature.

Service description enables composition through the definition of service interface. Interfaces dictate how the service can be accessed and controlled. A new service can be created by using the exposed interfaces of services already present in the network. It will have a set of sub-services and its own interface.

To enable discovery, it is necessary to describe the service through its name, type and other attributes. The service request message contains a partial description which is sent out to the network by the requesting entity. Nodes have to match the oncoming description with the one stored in the local memory to decide whether the service can be provided.

Service policy specifies which are the entities in the system that can access the service; more precisely, the service consumers. They can be just enumerated, or they can be identified based on certain characteristics specified within the service description.

Finally, the usage of the service is done through the exposed service interface, defined in the service description.

\section{SERVICE DESCRIPTION}

Our notation for service description is based on XML, which provides a set of guidelines and conventions for structuring and representing data. It is generic and easily extensible and it is popular with $\mathrm{W} 3 \mathrm{C}$ [11] as a means for structured information exchange and collaboration. By using XML as a building block for our description language, it is possible to take advantage of various publicly available open-source tools designed to work with XML.

CODE implements an XML schema which defines the elements that can appear in the description document. The key features of the schema are:

1. Attributes are not defined, so as to impose strict ordering of elements. This facilitates the implementation of efficient algorithms for tree traversal of descriptions.

2. The schema allows partial descriptions of elements. In this way, only important aspects can be expressed while keeping the size of the description to a minimum.

3. CODE allows every entity and service in the system to have application-specific attributes.

4. Entities can be identified based on context information (e.g. neighbours).

5. The schema can operate with variables that denote entities in the system and take values according to the context.

There are two components that can be described using CODE: (1) services and (2) entities. An entity is a device or a group of devices. An entity can play the role of service provider and service consumer.

A device is described in terms of the hardware, software, dynamics (fixed or mobile), location, groups that it is member of, connected entities (context information such as neighbours) and services it offers. A special type of device is the sensor, capable of measuring environmental conditions. Sensors are characterised by the type of measurements they perform and by the quality of service (range, resolution, accuracy). Groups are described by the number of members, the entity which is the initiator of group, the group members, the leader and the offered services.

Services are divided in two categories: monitoring and non-monitoring. Non-monitoring services are similar to an instantaneous function performed by the service provider, such as the average temperature measured by a group of nodes. They usually return a value as a result of a measurement or computation. Monitoring services perform timely supervision of a phenomenon, they are controlled through commands and issue events.

CODE defines the interface of a service, composed of inputs, outputs, commands and events. Moreover, it 
characterizes the service consumers, the sub-services, the requirements for competent service providers and the attributes of the service.

The following subsections focus on the challenges addressed by service management in $\mathrm{WCO}$, in particular the description of dynamic groups, on-demand services, service interface and sub-services.

\section{A. Groups}

Groups can be dynamically formed depending on the context. Context is defined by specifying a certain attribute, such as location, neighbouring devices or another parameter defined by the application.

Taking as example the scenario described in the previous section, beacons being in range of a cart form a group and provide a localization service to the cart. The cart for which the service is provided is known only at the moment it sends a service request. This cart is called the initiator of the group and its identity is not specified at the moment of service deployment. Instead, a variable is used to identify the cart, as shown in the following example:

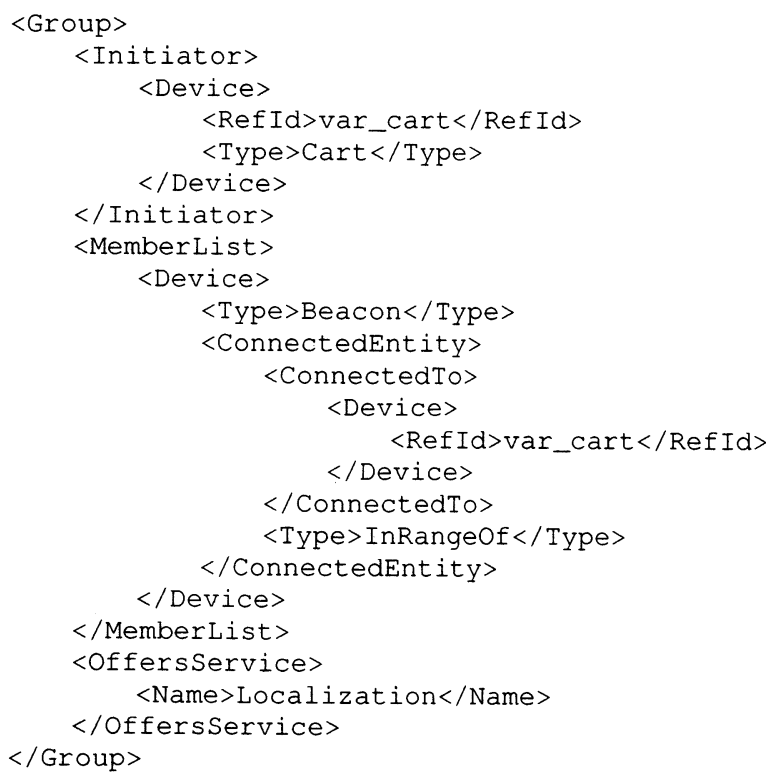

The description of the group contains the field Initiator, which designates the cart requesting localization service. Devices which are members of the group are the Beacons, with the restriction that they are inRangeOf the cart. The group offers the service named Localization. In the initialization phase, the variable denoting the cart is replaced with the appropriate cart ID.

\section{B. On-demand services}

CODE allows a service to be configured for being offered ondemand. The service requirements identify the potential providers of the service. In case that the provider is a group dynamically formed as a result of a service discovery request, the requirements specify the group characteristics. Here is a partial description of the localization service that uses the group description as requirements for service provider:

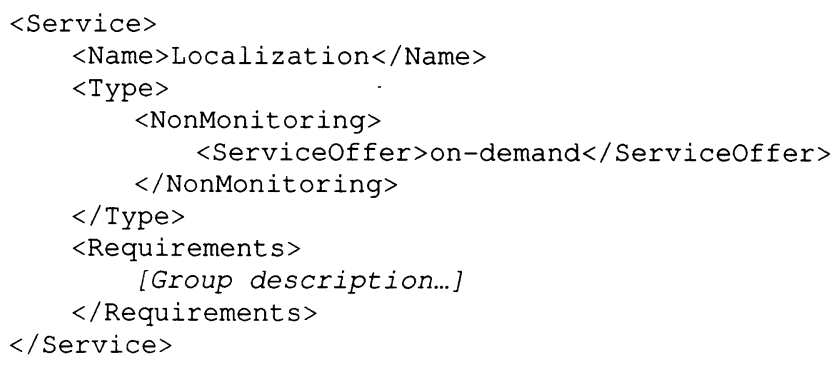

\section{Service interface}

The service interface is composed of the following entities:

- ParameterList - Service parameters can be:

$\circ$ the input information, compulsory for service initialization

- the output data, provided to the service consumer at the end of service utilization

- EventList - Events are messages sent to the service consumer to specify a state of the service. CODE defines the following categories of events: error, warning, alert, information and application defined.

- CommandList - Commands are control messages issued by the service consumer. They can trigger a previously defined event or they can query the service for a specific piece of information.

A simple interface for localization service defines an output parameter, representing the calculated position of the cart:

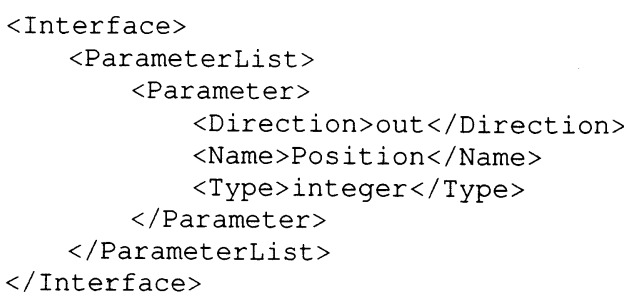

\section{Service policy}

Service policy consists of a set of entities which have the right to use the service. CODE allows specification of service policy by defining the characteristics of service consumers. Consumers do not need to be fully identified; rather they are characterised by an attribute or by the context. For example, a policy may state that entities which are allowed to access the temperature service are those located a maximum two hops away from CartX.

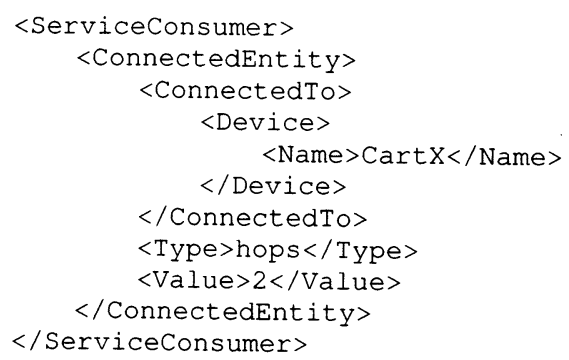




\section{E. Sub-services}

By means of interface definition, complex services can be developed from the existing ones. CODE allows the definition of sub-services which are a result of service composition. We take as an example the monitoring of carts gathering service, described in the transport scenario. Monitoring of carts uses the location service offered by each cart, which has to be delivered to the specified shop.

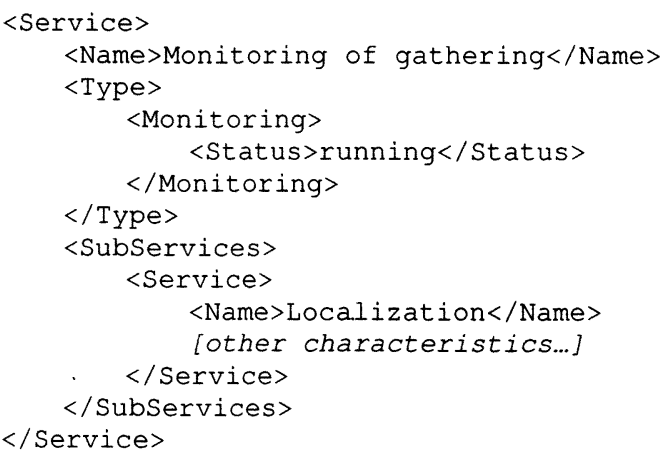

\section{IMPLEMENTATION ON SENSOR NODES}

CODE descriptions are based on XML, which provides flexibility and richness but is quite expensive in terms of memory usage and the processing power needed for parsing. This is the reason why the original XML descriptions are stored at the gateway level, while on sensor nodes we have implemented a binary XML version. The binary CODE preserves XML flexibility and extensibility, while optimizing for low storage and fast processing, as required by the hardware limitations of sensor nodes. Firstly, we will present the platform on which the tests have been performed. Secondly, we will analyse the storage space needed for binary CODE descriptions. Thirdly, we will show the processing time needed for matching a service discovery message to the service description stored on the node.

\section{A. Platform}

The implementation has been achieved on a hardware platform developed by NEDAP for the Eyes project [7]. It consists of MSP430f149 micro-controller from Texas Instruments that operates at $4 \mathrm{MHz}$. It has $60 \mathrm{kB}$ of program flash memory and $2 \mathrm{kB}$ of RAM. Other features include a radio transceiver and an $\mathrm{RS} 232$ interface. The operating system used is DCOS (Data Centric Operating System) [8], which occupies $5 \mathrm{kB}$ out of $60 \mathrm{kB}$ of flash memory.

\section{B. Binary descriptions}

The XML schema which defines CODE is used as the basis for the binary encodings. We have used the Java API for XML Processing (JAXP) [9] for parsing the XML descriptions through the Document Object Model (DOM) [10] interface. The XML DOM views XML documents as a tree structure of elements embedded within other elements. From this tree structure, we extract only the sub-tree containing the necessary information, leaving out the text descriptions and comments. The nodes in our tree can be
TABLE 1: IMPLEMENTATION RESULTS

\begin{tabular}{|l|l|l|l|l|}
\hline $\begin{array}{c}\text { Service } \\
\text { name }\end{array}$ & $\begin{array}{c}\text { Size of } \\
\text { uncompressed } \\
\text { description }\end{array}$ & $\begin{array}{c}\text { Size of } \\
\text { binary } \\
\text { description }\end{array}$ & $\begin{array}{c}\text { Number } \\
\text { of tree } \\
\text { nodes } \\
\text { (E+T) }\end{array}$ & $\begin{array}{c}\text { Matching } \\
\text { time of } \\
\text { binary } \\
\text { description }\end{array}$ \\
\hline Localization & $1.65 \mathrm{kB}$ & $114 \mathrm{~B}$ & 57 & $7 \mathrm{~ms}$ \\
\hline $\begin{array}{l}\text { Temperature } \\
\text { monitoring }\end{array}$ & $2.58 \mathrm{kB}$ & $202 \mathrm{~B}$ & 101 & $9 \mathrm{~ms}$ \\
\hline $\begin{array}{l}\text { Monitoring } \\
\text { of gathering }\end{array}$ & $3.52 \mathrm{kB}$ & $296 \mathrm{~B}$ & 148 & $10 \mathrm{~ms}$ \\
\hline
\end{tabular}

element or text. Considering that the XML schema contains 51 element types, we used one byte for encoding, thus leaving space for further extensions. We have pre-defined 5 types of text nodes: string, byte, int_16, int_32 and float. We have also decided to encode the strings, as the storage space is very limited. The resulting tree is further linearized, in depth first tree traversal order. Considering that we have $E$ number of element nodes and $T$ number of text nodes, the number of bytes $M$ occupied by the binary description is $M=2 * E+x * T$, where $x$ is a number between 2 and 5 and represents the average space occupied by a text node, depending on its type. Table 1 shows the results of compression for three of the services described in Section 2. The monitoring of gathering service defines a complex interface that allows the Central System to fully control the service. It has 96 element nodes and 52 text nodes, resulting in a binary description of only 296 bytes, which is still small enough to be stored in RAM.

\section{Matching binary descriptions}

In order to support service discovery, each node in the network stores the descriptions of the services it offers. Incoming service discovery messages are only partial descriptions of the service and they have to be kept as small as possible to achieve fast transmission and low power consumption. To decide whether a service request is satisfied, we have implemented a matching algorithm between the general description and the partial one. Taking advantage of the fact that (1) the trees to be matched have the same root, so matching of nodes is done at the same tree level; (2) matching of branches goes until the level of leaves; and (3) the element nodes are placed in strict order, we have implemented an nonrecursive algorithm that traverses the logical trees of the two binary descriptions. The tree corresponding to the complete description is traversed in one direction, without returning, while the tree associated with the partial description is traversed back and forth, depending on the matching result. At each step of the traversal, we keep the current levels of the trees equal. The upper bound of the algorithm complexity is $O(N)$, where $N=E+T$ is the total number of nodes in the tree associated with the general description. The lower bound of the algorithm is $O(n)$, where $n$ is the total number of nodes in the tree associated with the partial description.

The results of the experiments are shown in Table 1. The implementation revealed an execution time of $7 \mathrm{~ms}$ for localization service, considering a general description with $N=57$ (41 element nodes and 16 text nodes, which represent the leaves of the tree) and a partial description with $n=15$ (12 
element nodes and 3 text nodes). The implementation of the matching algorithm occupies $1.5 \mathrm{kB}$ of flash memory.

\section{RELATED WORK}

The challenges that WCOs bring into light change the traditional vision of service discovery protocols [4-6]. These protocols allow discovery of services in networks where the only possible service providers are stand-alone devices. The notion of on-demand services does not exist, as the service offer does not depend on the context. Moreover, there is no support for service composition.

Nevertheless, two description languages have been proposed, focusing particularly on sensor networks: SensorML [1, 2] and TinyML [3]. SensorML provides an XML schema for defining the geometric, dynamic, and observational characteristics of a sensor in support of data discovery. It supports the processing and analysis of the sensor measurements, the geolocation of observed values and provides performance characteristics. TinyML describes sensor platforms, sensor fields (a collection of sensor nodes) and virtual devices (a group of sensor nodes that perform a certain task). Both SensorML and TinyML define the notion sensor group, which is composed of multiple sensors that operate together to provide a collective observation or related group of observations. Unfortunately, these languages do not describe services in a sensor network; they only concentrate on defining the characteristics of devices. Moreover, XML descriptions are placed on the external interface, not on the sensor nodes. In-network processing of sensor descriptions is not one of the goals of Sensor ML or TinyML.

\section{CONCLUSIONS}

This paper introduced CODE, a description language for Wireless Collaborating Objects (WCOs). WCOs improve the traditional model of wireless sensor networks by enabling complex services to be offered either by single nodes or by groups of collaborating nodes. The purpose of CODE is to support service management for WCO, more specifically service deployment, service composition, service discovery, service policy and service usage. CODE is based on XML, widely used for structured information exchange and collaboration. The XML descriptions are stored at the gateway level, while the sensor nodes store and process the binary version. The binary CODE preserves XML flexibility and extensibility, while optimizing for low storage and fast processing, as required by the hardware limitations of sensor nodes. We have shown that a binary description of service is small enough to be stored on sensor nodes with just $2 \mathrm{kB}$ of RAM. Moreover, we have implemented an algorithm that matches a partial description against the complete one, thus providing the response to a service discovery messiage. The processing time is of the order of milliseconds, which is negligible in comparison with the communication overhead.

Future work will include extending the matching mechanism by allowing more complex validations, such as ränge checking. This will clearly be useful for practical situations; for instance the case of requesting a localization service with accuracy over a certain degree. We will also add functionality to the manipulation of binary descriptions, such as updating variables or changing fields which depend on context. Additionally, we will explore architectural choices for building efficient service discovery mechanisms on top of CODE descriptions.

\section{REFERENCES}

[1] Sensor Model Language (SensorML), 2004. http://vast.nsstc.uah.edu/SensorML/

[2] M. Botts (ed.) "Sensor Model Language (SensorML) for In-situ and Remote Sensors", OpenGIS Interoperability Program Report, OGC 04-019r2, Version 1.0.0 beta, Nov. 2004, http://vast.nsstc.uah.edu/SensorML/SensorML_04019_1.0_beta.pdf

[3] Nathan Ota and William T.C. Kramer, "TinyML: Meta-data for Wireless Networks", http://kingkong.me.berkeley.edu/ nota/research/Tiny $\mathrm{ML} /$ project-paper-1.pdf

[4] UPnP Forum, "UPnP device architecture", Version 1.0, June 2000, http://www.upnp.org/

[5] Sun Microsystems, "Jini architecture specification" version 2.0, June 2003, http://www.sun.com/software/jini/specs/jini2_0.pdf

[6] E. Guttman, C. Perkins, J. Veizades, and M. Day, "Service Location Protocol, Version 2," IETF, RFC 2608, June 1999, http://www.rfceditor.org/rfc/rfc2608.txt.

[7] Energy-Efficient Sensor Networks (EYES), http://www.eyes.eu.org/

[8] T.J. Hofmeijer, S.O. Dulman, P.G. Jansen and P.J. Havinga, "DCOS, a Real-Time Light-Weight Data Centric Operating System", In ACST 2004.

[9] Sun Microsystems, Inc., Java API for XML Processing (JAXP) 1.1 Public Review 2. http://java.sun.com/aboutJava/communityprocess/revi ew/jsr063/jaxp-pd2.pdf.

[10] A. L. Hors, P. L. Hegaret, L. Wood, G. Nicol, J. Robie, M. Champion and S. Byrne (Eds). "Document Object Model (DOM) Level 3 Core Specification" Version 1.0, W3C Recommendation, Apr. 2004, http://www.w3.org/TR/DOM-Level-3-Core/

[11] W3C World Wide Web Consortium,
http://www:w3.org/ 